\title{
EL MEJORAMIENTO DE LA COMPRENSIÓN DEL TEXTO DESDE UNA PERSPECTIVA COMPONENCIAL: EL CASO DE LA CAPACIDAD DE INDIVIDUALIZAR PERSONAJES, LUGAR Y TIEMPO
}

\section{TEXT COMPREHENSION IMPROVEMENT FROM A COMPONENTIAL PERSPECTIVE: THE ABILITY TO IDENTIFY CHARACTERS, PLACE AND TIME}

\author{
Chiara Meneghetti \\ Barbara Carretti \\ Rossana De Beni \\ Cesare Cornoldi \\ Università degli Studi di Padova, Italia \\ Valeria Abusamra \\ Universidad de Buenos Aires, Argentina
}

\begin{abstract}
Resumen: El objetivo del presente trabajo fue verificar la eficacia de un entrenamiento sobre la habilidad de base de identificar "personajes, lugares y tiempos" (PLT) utilizando una prueba de evaluación inicial, una de evaluación final y el programa de tratamiento propuesto en la Nuova Guida alla Comrpensione del Testo (De Beni et al., 2003a), posteriormente adaptada al español por Abusamra y colaboradores (en prensa). Participaron de esta investigación 119 estudiantes de tercero (43), cuarto (30) y quinto grado (46) de escuelas primarias de los cuales 59 fueron incluidos en el grupo control y 60 en el grupo experimental. Todos fueron evaluados con pruebas estandarizadas de comprensión de textos y con la prueba específica de PLT antes y después del entrenamiento. El entrenamiento tuvo una duración aproximada de cuatro meses y fue implementado con una frecuencia semanal tomando como base la ficha de PLT. Los resultados evidenciaron un efecto beneficioso de la intervención en todos los grupos experimentales. Dicha intervención resultó particularmente eficaz en el grupo experimental de $5^{\circ}$ grado que mostró no solo una mejoría específica en la prueba PLT sino además una generalizada en la prueba final que medía la comprensión (prueba MT).
\end{abstract}

Palabras clave: comprensión lectora, entrenamiento, mejoramiento.

\begin{abstract}
The aim of the present study was to verify the effectiveness of a training program based on the ability to identify "Characters, places and times" (CPT) using an initial evaluation test, a final evaluation test, and the treatment program proposed in Nuova Guida alla Comprensione del Testo (De Beni et al., 2003a), later adapted into Spanish (Abusamra et al., in press).119 students of third (43), fourth (30) and fifth grade (46) of primary schools participated in the reasearch. 59 of them were assigned to the control group and 60 to the experimental group.

All of them were evaluated with standardized text comprehension tests as well as with the specific CPT test before and after training took place. The training was implemented in approximately four month's time and was implemented on a weekly basis. Results demonstrated beneficial effects of the intervention in all the experimental groups. This intervention was particularly effective in the experimental group composed by school children of 5th grade, which exhibited not only specific improvement in the CPT test, but also general improvement in the comprehension test administered subsequently.
\end{abstract}

Keywords: reading comprehension, training, improvement.

\section{INTRODUCCIÓN}

Como ha sido demostrado por proyectos internacionales de evaluación (OCSE, PISA, etc.), la habilidad de comprensión del texto escrito constituye una de las competencias fundamentales a la cual todos los países del mundo aspiran. Lamentablemente, aun son muchos los estudiantes que no logran alcanzar estándares mínimos, con la consecuencia de 
tropezar con dificultades para procesar distintas formas de texto escrito. Un desafío que se suscita para quienes estudian el aprendizaje está en entender por qué aparecen dificultades en la comprensión de textos y cómo se puede intervenir para revertir las dificultades o mejorar dicha capacidad. Este desafío alcanza también a quienes se ocupan de las alteraciones de lectura (v. Shaywitz, Morris \& Shaywitz, 2008) y revela que existe una desproporción entre el tratamiento de las habilidades de decodificación de palabra aislada y el tratamiento de la habilidad de comprensión.

Si bien es verdad que los déficits lingüísticos representan un rasgo común de los estudiantes con alteraciones de la comprensión lectora, ha sido, sin embargo, demostrado que los sujetos con alteraciones de la comprensión de textos se caracterizan por exhibir perfiles extremamente variables (Cain e Oakhill, 2006; Cornoldi, De Beni e Pazzaglia, 1996). Cornoldi et al. (1996), examinando y siguiendo longitudinalmente durante tres años a un grupo de estudiantes de 11 años con trastornos de comprensión de textos, encontraron dichas desigualdades en el rendimiento diferencial de los estudiantes.

Algunos planteaban dificultades en la comprensión de mensajes presentados oralmente, mientras que para otros, los déficits en el conocimiento general, en la memoria de trabajo o en el control metacognitivo constituían un factor relevante.

Aun teniendo en cuenta las competencias intelectuales generales (medidas con el PMA verbal y de razonamiento), Cornoldi et al (1996) notaron la misma variabilidad de perfiles, con estudiantes con buenas actuaciones en ambos subtest de la batería y otros en los que solo una o ambas actuaciones resultaban deficitarias. Recientemente, Cain y Oakhill (2006) reportaron resultados similares.

En particular, Cain y Oakhill (2006) analizaron el perfil de un grupo de niños de 7 y 8 años con dificultades de comprensión de textos en pruebas de memoria de trabajo, de funcionamiento general (e.g. IQ), de conocimiento léxico (e.g. pruebas de vocabulario), de comprensión general del texto (e.g. la Neal Reading Comprehension, que es la prueba de comprensión estandarizada, utilizada en Inglaterra), pruebas que medían aspectos más específicos (e.g. capacidad de generar inferencias o individualizar la estructura narrativa del texto) y pruebas de velocidad de lectura.
De los resultados obtenidos, surge que las competencias cognitivas generales no determinan las diferencias en la actuación en pruebas de comprensión (contrariamente a lo evidenciado por Nation et al. 2002), aunque predicen la evolución de la alteración de comprensión: estudiantes con un funcionamiento general más bajo (pero en los límites de la norma) muestran una capacidad de mejoramiento menor en pruebas de comprensión del texto a los 11 años. Además, el análisis de los perfiles individuales de los estudiantes con alteraciones de comprensión mostró la misma variabilidad evidenciada en el estudio de Cornoldi et al (1996) con puntos de fuerza y debilidad diferentes según el caso.

En la comprensión del texto están implicados diferentes procesos cognitivos subyacentes, entre los cuales puede destacarse la memoria de largo plazo (que almacena los conocimientos que el lector posee), la atención y la memoria de trabajo. Es por esto que entre las muchas propuestas presentes en la literatura que intentan explicar cómo se comprende un texto, todos los modelos acuerdan en el hecho de que la comprensión del texto es un proceso activo de construcción del significado que depende no sólo de la información textual explícita sino además de la información que posee el lector (ver por ejemplo, Johnson-Laird, 1983 o Kintsch y Van Dijk, 1978). Incluso, estudios clásicos de la psicología cognitiva (Bransford e Johnson, 1972) han, de hecho, señalado que cuando no se produce esta interacción entre la información presente en el texto y el conocimiento previo, el lector, aun teniendo la posibilidad de comprender el significado del texto a un nivel de superficie (es decir, un nivel de significado en el que se priorizan los significados de las palabras individuales, conocido como nivel del textobase, ver van Dijk y Kintsch, 1983), no logra acceder al significado global y, por lo tanto, no alcanza a construir un modelo mental o situacional coherente del contenido del texto.

Son muchas las perspectivas que han tratado de establecer un elenco sistemático de elementos fundamentales comprometidos en la comprensión del texto escrito. Uno de éstas, ha llevado a la identificación de 10 componentes (De Beni et al., 2003a, Carretti, Meneghetti y De Beni, 2005) (ver Tabla 1), en función de los cuales se construyeron pruebas de evaluación y se diseñaron programas de intervención. Posteriormente, un grupo de la Universidad de Buenos Aires ha llevado a cabo la adaptación 
a la lengua española, incorporando a las diez áreas originales una nueva: Semántica léxica (Abusamra et al., 2007; en prensa).

Tabla 1. Taxonomía de la Nueva Guía para la Comprensión (ver De Beni et al, 2003a)

\begin{tabular}{|c|c|}
\hline $\begin{array}{c}\text { Personajes, lugares, tiempos } \\
\text { y hechos }\end{array}$ & Sensibilidad al texto \\
\hline Hechos y secuencias & Jerarquía del texto \\
\hline Estructura sintáctica & Modelos mentales \\
\hline Cohesión & Flexibilidad \\
\hline Inferencias & Errores e incongruencias \\
\hline
\end{tabular}

En la actividad clínica, resulta poco práctica la utilización de todos los componentes para orientar el posible tratamiento. La elección de las áreas necesarias para profundizar en un trastorno de comprensión, podría ser efectuada a través del análisis de las respuestas dadas por el niño en un screening de carácter general, que podría dar indicaciones sobre el aspecto que es importante enfatizar.

A diferencia de lo que ocurre en la clínica, en un proyecto educativo a largo plazo, llevado a cabo en el marco de la escuela, la atención puede ser puesta (si no en todos) en muchos componentes. En algunos cursos, la valoración de la totalidad de los componentes permitirá un diagnóstico general más certero que ponga en evidencia ciertas especificidades del grupo y guíe la elección posterior de pruebas de profundización. La idea subyacente es que para construir una habilidad compleja como lo es la comprensión de un texto puede ser ventajoso operar en paralelo, pero de manera independiente, sobre algunos de los componentes del proceso.

En los últimos años, numerosas reseñas han intentado establecer, a través de una variedad de tratamientos propuestos, la eficacia de un procedimiento de intervención respecto de otros (Mastropieri y Scruggs, 1997; Pazzaglia y Rizzato, 2001; Gajria et al., 2007). Pazzaglia y Rizzato (2001) han distinguido tres tipos de programas para la promoción de las habilidades de comprensión textual:

1. intervenciones de tipo cognitivo que apuntan a la enseñanza de estrategias específicas;

2. tratamientos que integran la enseñanza de estrategias con la promoción de competencias cognitivas y metacognitivas relativas al texto y a la lectura y

3. tratamientos que asocian la instrucción sobre estrategias y conocimiento metacognitivo con aspectos motivacionales y atributivos implicados en el aprendizaje. En concordancia con los resultados del meta análisis realizado por Swanson y Hoskyn (1998), Pazzaglia y Rizzato (2001) revelaron que los programas más efectivos son aquellos que, después de un tratamiento de tipo estratégico (que instruye al niño sobre el uso de estratégicas específicas), promueve habilidades de tipo cognitivo y metacognitivo.

La inclusión de elementos metacognitivos favorece que el alumno adquiera nuevas estrategias, que reflexione sobre su utilidad, que mejore su propio conocimiento sobre los objetivos de la lectura y que intensifique la habilidad de control (ver por ejemplo Miranda, Vilalescusa y Vidal-Abarca, 1997). La promoción de estas reflexiones - dada la generalidad de la adquisición - origina cambios, también, en otros ámbitos cognitivos como demostraron Lucangeli, Galderisi y Cornoldi (1994) en su investigación.

En particular, los nuevos materiales para el tratamiento incluidos en el proyecto Nuova Guida alla Comprensione del Testo están compuestos por tres volúmenes pensados para edades diferentes (volumen 2 De Beni, Cornoldi, Caponi y Gasparetto, 2004: primero años de la escuela primaria; volumen 3 De Beni, Vocetti, Cornoldi y Gruppo MT, 2003b: $3^{\circ}$ a $7^{\circ}$ grado; volumen 4 De Beni, Vocetti, Cornoldi y Gruppo MT, 2003b: $7^{\circ}$ grado a $2^{\circ}$ año de escuela secundaria).

Las áreas de tratamiento están organizadas de acuerdo con la taxonomía propuesta para las pruebas de evaluación (De Beni et al., 2003a), previendo, de esta manera, la realización de actividades específicas sobre alguna habilidad cognitiva en particular (como por ejemplo, generar inferencias, individualizar personajes, lugares y tiempos en una historia) o actividades que trabajen sobre los aspectos metacognitivos ligados a la comprensión (como por ejemplo, conocimiento y utilización flexible de estrategias de comprensión).

En ambos tipos de actividad, el estudiante es impulsado a reflexionar y a ejercitarse en las operaciones necesarias para llevar a cabo la tarea. 
Son muchas las experiencias que han demostrado los beneficios del uso de esta perspectiva basada en múltiples componentes, pero faltan estudios controlados que examinen los efectos que puede tener un tratamiento que focalice en un solo componente.

El presente estudio se propone justamente exponer una contribución en esta dirección.

Como la experimentación fue aplicada en niños de una edad relativamente baja, decidimos focalizar sobre una habilidad que es crítica en esta etapa y que está constituida por la capacidad de orientarse en las unidades fundamentales de una estructura narrativa, revelando las características esenciales relativas a personajes, lugares y tiempos.

\section{MÉTODO}

\section{Participantes}

Participaron de esta investigación 119 estudiantes de $3^{\circ}, 4^{\circ}$ y $5^{\circ}$ grado de escuela primaria.

En la Tabla 2 se explicita el número de alumnos de cada grado que formaron parte del grupo control y del experimental.

Tabla 2. Número de participantes de $3^{\circ}, 4^{\circ}$ y $5^{\circ}$ grado divididos por grupos experimental y control

\begin{tabular}{|c|c|c|c|}
\cline { 2 - 4 } \multicolumn{1}{c|}{} & $\begin{array}{c}\text { Grupo } \\
\text { control }\end{array}$ & $\begin{array}{c}\text { Grupo experi- } \\
\text { mental }\end{array}$ & Total \\
\hline Tercer grado & 22 & 21 & 43 \\
\hline Cuarto grado & 13 & 17 & 30 \\
\hline Quinto grado & 24 & 22 & 46 \\
\hline Totales & $\mathbf{5 9}$ & $\mathbf{6 0}$ & \\
\hline
\end{tabular}

\section{Materiales}

\section{Medidas utilizadas para evaluar el pre $y$ post tratamiento.}

a) Prueba MT inicial y final (Cornoldi y Colpo, 1998). Estas pruebas tienen el objetivo de evaluar la comprensión general del texto.

Fueron utilizadas seis pruebas, dos por cada clase; una fue administrada al inicio y la otra al final de la intervención. Cada una consistía en la lectura de un texto seguida de 12 preguntas con opciones de respuesta múltiple para $3^{\circ} \mathrm{y}$ $4^{\circ}$ grado y de 14 preguntas para $5^{\circ}$ grado. Los textos eran del tipo de material utilizado por los niños italianos de los últimos grados de la escuela primaria e incluían elementos tanto descriptivos como narrativos.

b) Pruebas para la evaluación de la habilidad "Personajes, lugares, tiempos y hechos" (PLT; De Beni et al., 2003a). Esta prueba tiene el objetivo de evaluar la capacidad específica de individualizar en un texto cuáles son los personajes, en que lugar(es) se desarrolla la acción y cuánto duran los hechos narrados. Esta habilidad está estrechamente ligada a la capacidad de encontrar y organizar temporalmente los eventos y hechos principales.

c) Material para el tratamiento. Se utilizó la ficha de tratamiento de Personajes - Lugares - Tiempos (PLT) propuesta por De Beni et al. (2003b). La ficha promueve la capacidad del estudiante de:

1. Para Personajes: individualizar los nombres, los sinónimos o definiciones con los que son indicados personajes en el texto; revelar los intercambios recíprocos, reconocer personajes principales y secundarios; distinguir entre personajes reales/verosímiles y de fantasía; individualizar personajes representantes de una categoría; tomar el punto de vista de los diversos personajes.

2. Para Lugares: detectar los lugares citados en el texto, inferir lugares no citados explícitamente, organizar lógicamente los lugares detectados; distinguir entre lugares reales/ verosímiles y de fantasía.

3. Para Tiempos: identificar la época de la acción y la duración de la misma; inferir sobre ambos aspectos, distinguir épocas reales e imaginarias (pasado y futuro); registrar contemporaneidad, anterioridad y posterioridad de las acciones.

4. Para Hechos: individualizar los hechos de un texto y distinguir los principales de los secundarios; distinguir entre hechos reales/ verosímiles y de fantasía.

5. Para Géneros textuales: establecer diferencias entre género narrativo y no narrativo; analizar personajes, lugares, tiempos y tipos de acciones computadas en los diversos géneros textuales. 


\section{Procedimiento}

El tratamiento para el grupo experimental tuvo una duración de aproximadamente 4 meses y fue iniciado en la mitad del año escolástico. Un psicólogo experto aplicó en las clases seleccionadas las pruebas MT inicial y la prueba criterial PLT siguiendo un procedimiento común en todos los grupos evaluados. El entrenamiento fue llevado a cabo por el mismo psicólogo con una frecuencia semanal, dos horas cada vez y en horario matutino. El esquema de entrenamiento fue aplicado colectivamente en cada clase. La modalidad general previó una fase individual o en pequeños grupos y una fase de discusión grupal.

A diferencia del grupo experimental, el grupo control fue estimulado con tareas de comprensión de acuerdo con los procedimientos típicos de la escuela que trabajan de modo simultáneo sobre todos los aspectos de la comprensión.

Cuando finalizó el tratamiento, se evaluó inmediatamente a todas las clases con la prueba MT final y la misma prueba criterial PLT.

\section{Diseño experimiental}

El diseño experimental previó tres factores: 2 factores entre los sujetos, es decir el factor clase en sus tres niveles $\left(3^{\circ}, 4^{\circ}\right.$ y $5^{\circ}$ grado) y el factor grupo en dos niveles (grupo experimental y control), y el factor "fase de evaluación" (pre y post tratamiento) como factor intrasujeto.

\section{RESULTADOS}

A los efectos del análisis fueron excluidos los estudiantes con retrasos en el desarrollo (4 de $3^{\circ}$ grado y 2 de $5^{\circ}$ ).

a) Prueba criterial PLT. Se llevó a cabo un análisis de varianza de medidas repetidas del tipo 2 (fase de medición: pre vs post tratamiento) $\times 2$ (grupo: experimental vs control) $\mathrm{x}$ 3 (clase: $3^{\circ}$ vs $4^{\circ}$ vs $5^{\circ}$ grado).

Los resultados evidenciaron un efecto principal del factor fase de medición con $\mathrm{F}(1,107)=28.74 \mathrm{MSE}=5.41 \mathrm{\eta} 2=.21 \mathrm{p} \leq .001$, por el cual la actuación en la prueba durante el post-tratamiento $(\mathrm{M}=8.86 \mathrm{ES}=.35)$, resultó superior respecto de la fase pre-tratamiento,
$(\mathrm{M}=7.17 \mathrm{ES}=.26)$. Además, se evidenció un efecto principal del factor clase $F(2,107)=$ 22.57 MSE $=15.00 \eta 2=.30 p \leq .001$.

La comparación posterior entre medias, aplicando la correlación por confrontación múltiple de Bonferroni puso de manifiesto que el rendimiento de $3^{\circ}(\mathrm{M}=6.34 \mathrm{ES}=$ $.44)$ y $4^{\circ}$ grado $(M=7.44 \mathrm{ES}=.51)$, no es significativamente diferente, pero si es significativamente inferior respeto al de $5^{\circ}$ grado $(\mathrm{M}=10.27 \mathrm{ES}=.41 \mathrm{p} \leq .001)$.

La interacción entre fase de medición $\mathrm{x}$ grupo resultó significativa $F(1,107)=7.25 \mathrm{MSE}=$ $5.41 \eta 2=.06 \mathrm{p} \leq .01$.

Las medias y los desvíos estándar de la prueba PLT del grupo experimental y del grupo control en las fases de pre y post-tratamiento se presentan en la Tabla 3.

Tabla 3. Medias y desvíos estándar en la prueba PLT de los grupos experimental y control en el pre y post tratamiento

\begin{tabular}{|c|c|c|c|}
\cline { 3 - 4 } \multicolumn{2}{c|}{} & $\begin{array}{c}\text { Pre - } \\
\text { tratamiento }\end{array}$ & $\begin{array}{c}\text { Post - } \\
\text { tratamiento }\end{array}$ \\
\hline \multirow{2}{*}{$\begin{array}{c}\text { Grupo } \\
\text { control }\end{array}$} & M & 7.38 & 8.15 \\
\cline { 2 - 4 } & ES & .45 & .56 \\
\hline $\begin{array}{c}\text { Grupo } \\
\text { experimental }\end{array}$ & M & 7.28 & 9.78 \\
\cline { 2 - 4 } & ES & .49 & .45 \\
\hline
\end{tabular}

Las comparaciones establecidas evidenciaron que la actuación del grupo control es igual en el pre y post-test con $t(54)=1.42 p=.16$, mientras que el grupo experimental tiene una actuación mejor en instancia posterior al tratamiento que en la fase anterior al mismo con $t$ $(54)=6.91 \mathrm{p} \leq .001$.

La interacción entre la fase de medición $x$ clase resultó significativa $\mathrm{F}(2,107)=6.37 \mathrm{MSE}$ $=5.41 \eta 2=.11 \mathrm{p} \leq .01$.

Las medias y los desvíos estándar de la prueba PLT de $3^{\circ}, 4^{\circ}$ y $5^{\circ}$ grado en el pre y posttratamiento se presentan en la Tabla 4.

Las comparaciones establecidas con el fin de comparar las actuaciones en la prueba PLT en el pre y post-tratamiento en cada clase mostraron que la performance en la prueba mejora en la etapa de post-tratamiento (respecto del pretratamiento) en $3^{\circ}$ grado, $\mathrm{t}(38)=5.65 \mathrm{p} \leq .001$ y en $4^{\circ}$ grado $t(29)=2 . .45 p=.02($ ver Tabla 3$)$. 
Mientras que no cambia significativamente en $5^{\circ}$ grado $(t<1)$ donde - se recordará - la actuación fue en general más elevada.

No resultó significativo el efecto de grupo $\mathrm{F}$ $=2.79 p=.10$, la interacción grupo $x$ clase ni la fase de medición $x$ grupo $x$ clase $(F<1)$.

El resultado de la interacción entre fase de medición x clase nos llevaría a concluir que hay un mejoramiento de ambos grupos (experimental y control) en $3^{\circ}$ y $4^{\circ}$ grado pero no en $5^{\circ}$.

Si observamos los estadísticos descriptivos de $5^{\circ}$ grado (ver Tabla 5 ) se observa que las medias en el pret-test del grupo experimental son un poco más altas que las del grupo control (aunque la diferencia no es significativa) y en el post-test, el grupo control presenta medias un poco más bajas respecto al pre-test contrastando la tendencia de $3^{\circ}$ y $4^{\circ}$ grado a mejorar en el post-test.

Tabla 4. Media y desvíos estándar en la prueba PLT de $3^{\circ}$, $4^{\circ}$ y $5^{\circ}$ grado en el pre y post tratamiento

\begin{tabular}{|c|c|c|c|}
\hline & & $\begin{array}{c}\text { Pre - } \\
\text { tratamiento }\end{array}$ & $\begin{array}{c}\text { Post - } \\
\text { tratamiento }\end{array}$ \\
\hline \multirow{2}{*}{$\begin{array}{l}\text { Tercer } \\
\text { grado }\end{array}$} & $M$ & 4.83 & 7.86 \\
\hline & ES & .44 & .58 \\
\hline \multirow{2}{*}{$\begin{array}{l}\text { Cuarto } \\
\text { grado }\end{array}$} & $M$ & 6.65 & 8.23 \\
\hline & ES & .50 & .67 \\
\hline \multirow{2}{*}{$\begin{array}{l}\text { Quinto } \\
\text { grado }\end{array}$} & $M$ & 10.04 & 10.49 \\
\hline & ES & .41 & .54 \\
\hline
\end{tabular}

Tabla 5. Medias y desvíos estándar de $3^{\circ}, 4^{\circ}$ y $5^{\circ}$ grado distinguiendo los grupos experimental y control en el pre y post tratamiento

\begin{tabular}{|l|c|c|c|c|c|}
\cline { 3 - 6 } \multicolumn{2}{c|}{} & \multicolumn{2}{c|}{ Grupo control } & \multicolumn{2}{c|}{ Grupo experimental } \\
\cline { 2 - 6 } \multicolumn{2}{c|}{} & $\begin{array}{c}\text { Pre - } \\
\text { tratamiento }\end{array}$ & $\begin{array}{c}\text { Post - } \\
\text { tratamiento }\end{array}$ & $\begin{array}{c}\text { Pre - } \\
\text { tratamiento }\end{array}$ & $\begin{array}{c}\text { Post - } \\
\text { tratamiento }\end{array}$ \\
\hline \begin{tabular}{|l|c|c|c|} 
Trerce \\
grado
\end{tabular} & $\mathrm{M}$ & 5.05 & 7.32 & 4.60 & 8.40 \\
\cline { 2 - 6 } & $\mathrm{ES}$ & .62 & .83 & .61 & .81 \\
\hline $\begin{array}{l}\text { Cuarto } \\
\text { grado }\end{array}$ & $\mathrm{M}$ & 6.77 & 7.46 & 6.53 & 9.00 \\
\cline { 2 - 6 } & $\mathrm{ES}$ & .75 & 1.00 & .66 & .88 \\
\hline $\begin{array}{l}\text { Quinto } \\
\text { grado }\end{array}$ & $\mathrm{M}$ & 9.65 & 9.22 & 10.43 & 11.76 \\
\cline { 2 - 6 } & $\mathrm{ES}$ & .54 & .79 & .56 & .83 \\
\hline
\end{tabular}

El curso de los estadísticos descriptivos explicaría el por qué en el pre y post-entrenamiento las medias de $5^{\circ}$ grado no diferían.

b) Prueba de comprensión MT. Es importante recordar que las pruebas de comprensión utilizadas son diferentes no solo para cada clase sino además - dentro de una misma clase - para cada instancia de evaluación (pre y post entrenamiento, con un aumento de dificultad).

Es por esto que la comparación entre los puntajes absolutos de las pruebas (cuya dificultad varía) no es informativa, pero sí es interesante observar si los grupos experimentales y controles, distinguidos por clase, tuvieron comportamientos diferentes al final del entrenamiento.

Se llevaron a cabo, entonces, tres análisis de varianza $2 \times 2$ (uno por cada clase) para ver si se encontraba una interacción entre grupos y la comparación pre y post, debida al hecho de que el grupo experimental se había beneficiado en el nivel de comprensión general. Para $5^{\circ}$ grado la interacción entre fase de medición y grupo resultó significativa $\mathrm{F}(1,42)=4.03 \mathrm{MSE}$ $=4.14 \eta 2=.09 p=.05$. Las comparaciones realizadas indicaron que la actuación en la prueba de comprensión del grupo control es peor en la evaluación final $(\mathrm{M}=7.52 \mathrm{ES}=.74)$ en comparación con la evaluación inicial $(\mathrm{M}=9.21$ $\mathrm{ES}=.65)$ con $\mathrm{t}(22)=2.49 \mathrm{p}=.02$, por la mayor dificultad de la prueba prevista para el final. A pesar de la dificultad, el grupo experimental logró afrontar también adecuadamente la prueba final. La actuación del grupo experimental es igualmente adecuada antes $(\mathrm{M}=10.04 \mathrm{ES}=$ .77) y después del tratamiento $(\mathrm{M}=10.00 \mathrm{ES}=$ .68) con $t<1$.

\section{DISCUSIÓN}

El objetivo de la presente investigación fue verificar la eficacia de un entrenamiento sobre una habilidad de base de la comprensión de textos que consiste en identificar y reconocer personajes, lugares y tiempos de una narración.

El entrenamiento, aplicado en $3^{\circ}, 4^{\circ}$ y $5^{\circ}$ grado, resultó completamente eficaz y dicha eficacia se evidenció, sobre todo, en el mejoramiento de la capacidad específica PLT de los niños del grupo que habían sido estimulados. Esto fue confirmado por la interacción fase de medición x grupo: en el grupo control, la actuación en la prueba específica (es decir la de PLT) resultó similar en la primera y segunda evaluación; en el grupo experimental, en cambio, la actuación resultó significativamente mejor en la segunda evaluación que en la primera: de 
hecho, la precisión en la prueba PLT resultó superior en el post-entrenamiento que en el pre-entrenamiento.

Es importante plantear algunas consideraciones diferenciales para cada grado. En $3^{\circ}$ y $4^{\circ}$ grado - que tuvieron en general un rendimiento inferior a $5^{\circ}$ grado - todos los estudiantes (tanto los del grupo experimental como los del grupo control) demostraron mejores actuaciones en la evaluación post-entrenamiento que en la del pre-entrenamiento. Esto puede interpretarse en relación con los lineamientos escolares de $3^{\circ} \mathrm{y}$ $4^{\circ}$ grado donde hay aspectos de base de la comprensión como el que se trabajó en este caso que son parte de los programas curriculares.

A diferencia de lo que ocurre en los grados inferiores, el rendimiento en $5^{\circ}$ grado fue distinto y la eficacia del entrenamiento parece ser específica. Si bien el grupo experimental de $5^{\circ}$ grado parte con una ventaja inicial respecto del grupo control, el primero es quien se beneficia del entrenamiento. Mientras el grupo control mantiene un rendimiento similar en la prueba PLT en la evaluación inicial y final, el grupo experimental logra mayor adecuación de respuesta en la prueba luego de haber sido sensibilizado con el entrenamiento.

¿Puede este resultado extenderse también a los obtenidos en la prueba de comprensión MT que valora la habilidad general de comprensión?

En este punto, sería ambicioso esperar resultados extremadamente relevantes ya que se ha trabajado sobre un solo componente de la comprensión. No obstante, se encontró una ventaja del grupo experimental, sobre todo en $5^{\circ}$ grado, para el cual, aparentemente el beneficio en la habilidad específica PLT fue menos evidente (tal vez por el hecho de que la competencia era buena aun antes de la intervención). De hecho, mientras el grupo control empeora en la evaluación final con la prueba MT respecto de la inicial, el grupo experimental mantiene estable el rendimiento.

Como dijimos anteriormente, acordando con los resultados del meta análisis llevado a cabo por Swanson y Hoskyn, (1998), Pazzaglia y Rizzato (2001) revelaron, en primer término, que la habilidad de comprensión del texto puede ser sensiblemente mejorada mediante procedimientos idóneos; en segundo lugar, que son particularmente eficaces los programas que, a la par de un tratamiento de tipo estratégico (que instruye al niños sobre el uso de estrategias específicas) promueven habilidades de tipo cognitivo y metacognitivo. Los resultados de la presente investigación parecen proveer evidencia convergente en favor de esta conclusión.

El trabajo sobre un componente cognitivo particular de la comprensión de textos, con la inclusión de estimulación metacognitiva (como fue previsto en el programa de De Beni y colaboradores, 2003b) ha influido sobre la habilidad de comprensión. Es posible que la integración de este trabajo con actividades que entrenen de manera más directa un componente metacognitivo pueda llevar a un mejoramiento del grupo experimental aun más evidente.

\section{REFERENCIAS}

Abusamra, V., Ferreres, A., Raiter, A., De Beni. R. \& Cornoldi, C. (en prensa). Leer para comprender: Test para evaluar la comprensión de textos. Buenos Aires: Editorial Paidos.

Abusamra, V., Casajús, A., Cartoceti, R. Zunino, G. Miranda, A., Badía, J., Ferreres, A., Raiter, A., De Beni. R. \& Cornoldi, C. (2007). "Evaluación de la comprensión de textos con un modelo de múltiples componentes". X Congreso de la Sociedad Latinoamericana de Neuropsicología (SLAN). Buenos Aires. Argentina. 7 al 10 de noviembre.

Bransford, J. D., \& Johnson, M. K. (1972). Contextual prerequisites for understanding: some investigations of comprehension and recall. Journal of Verbal Learning and Verbal Behaviour, 11, 717-726.

Cain, K. (2006). Individual differences in children's memory and reading comprehension: an investigation of semantic and inhibitory deficits. Memory, 14, 553-569.

Cain, K., \& Oakhill, J. (2006). Profiles of children with reading comprehension difficulties. British Journal of Educational Psychology, 76, 683-696.

Carretti, B., Meneghetti, C., \& De Beni, R. (2005). Evoluzione delle abilità di comprensione in studenti dalla 3a elementare alla 1a media. Età Evolutiva, 80, 5-16.

Cornoldi,C., \&Colpo, G.(1998). ProvediletturaMTperlascuola elementare 2, Firenze: Organizzazioni Speciali.

Cornoldi, C., De Beni, R., \& Pazzaglia, F. (1996). Profiles of reading comprehension difficulties: An analysis of single cases. In C. Cornoldi \&J. Oakhill, (Eds.), Reading comprehension difficulties: Processes and intervention (pp. 113-136). Mahwah, NJ: Lawrence Erlbaum Associates, Inc. 
De Beni R., Cornoldi C., Carretti B. \& Meneghetti C (2003a). Nuova guida alla comprensione del testo. Volume 1. Introduzione teorica generale al programma. Le prove criteriali livello A e B. Trento, Erickson. pp. 191.

De Beni R., Vocetti C., Cornoldi C. \& Gruppo MT (2003b). Nuova guida alla comprensione del testo. Volume 3 e Volume 4. Trento, Erickson.

De Beni, R., Cornoldi, C., Caponi, B., \& Gasparetto, R. (2004). Nuova Guida alla Comprensione: Volume 2. Trento: Erickson.

Gajria, M; Jitendra, A. K; Sood, S. \& Sacks, G. (2007). Improving comprehension of expository text in students with LD: A research synthesis. Journal of Learning Disabilities, 40 (3), 210-225.

Johnson-Laird, P.N.(1983). Mentalmodels: Towardsacognitivescience oflanguage, inference, and consciousness. Cambridge, MA: Harvard University Press.

Kintsch, W., \& van Dijk, T. A. (1978). Toward a model of text comprehension and production. Psychological Review, 85, 363-394.

Lucangeli, D., Galderisi, D., \& Cornoldi, C. (1995). Specific and general transfer effects following metamemory training. Learning Disabilities Research and Practice, 10, 11-21.

Mastropieri, M.A., \& Scruggs, T.E. (1997) Best practices in promoting reading comprehension in students with learning disabilities: 1976 to 1996 . Remedial and Special Education, 18, 197-213.
Meneghetti, C., Carretti, B. \& De Beni R. (2007). Components of reading comprehension and scholastic achievement. Learning and Individual Differences. doi:10.1016/j.lindif.2006.11.001.

Miranda, A., Villaescusa, M. I., \& Vidal-Abarca, E. (1997). Is attribution retraining necessary? Use of self-regulation procedures for enhancing reading comprehension strategies of children with learning disabilities. Journal of Learning Disabilities, 30, 503-512.

Nation, K., Clarke, P., \& Snowling, M. J. (2002). General cognitive ability in children with reading comprehension difficulties. British Journal of Educational Psychology, 72, 549-560.

Pazzaglia, F., \& Rizzato, R. (2001). Trattamenti metacognitivi per migliorare la comprensione della lettura: quale efficacia? Età Evolutiva, 68, 104-117.

Shaywitz, S. E; Morris, R \& Shaywitz, B. A. (2008). The education of dyslexic children from childhood to young adulthood. Annual Review of Psychology, 59, 451-475.

Swanson, H. L., \& Hoskyn, M. (1998). Experimental intervention research on students with learning disabilities: A meta-analysis of treatment outcomes. Review of Educational Research, 68, 277-321.

Van Dijk, T. A., e Kintsch, W. (1983). Strategies of discourse comprehension. New York: Academic Press. 